\title{
Spontaneous recovery without interference: Why remembering is adaptive
}

\author{
LYNN D. DEVENPORT \\ University of Oklahoma, Norman, Oklahoma
}

\begin{abstract}
Spontaneous recovery from extinction is a reliable result of two ingredients, variable training outcomes and the passage of time. Accounts of the phenomenon, which have come to focus on interference at the level of memory retrieval, have been based on simple associative learning tasks. The present study was designed to determine whether a more complex task-one requiring spatial mapping, timing, and patch assessment-would be subject to spontaneous recovery. Results showed that spontaneous recovery (1) makes a robust appearance in such tasks, (2) does not require interference among conflicting memories, but instead (3) requires ready access to training memories, and (4) is closely modeled by the temporal weighting rule, a quantitative model of patch choice that prescribes how value is best assigned to variable resources (L. D. Devenport \& J. A. Devenport, 1994).
\end{abstract}

What happens when reward is withdrawn is an enduring theoretical difficulty. Objectively, extinction diminishes the rate or vigor of behavior and eventually causes it to cease altogether. The most parsimonious explanations held that extinction is equivalent to the undoing of excitatory associations (e.g., Rescorla \& Wagner, 1972). Dogging this and any other account of conditioning as a single bond that can be strengthened or loosened is spontaneous recovery (SR), the almost inevitable reemergence of an extinguished response with the passage of time, showing that the original association was not unlearned, nor its memory erased. SR has stood for decades as a conundrum for a discipline devoted to explaining behavior in terms of associative learning because an apparently nonassociative process so consistently and dramatically alters behavior (reviewed in Bouton, 1993; Robbins, 1990). Pavlov (1927) seemed to attribute the effects of extinction to diminished attention to the conditioned stimulus (CS), and this was the only view for which Robbins could find empirical support. Unfortunately, neither Pavlov nor Robbins could explain why processing of the CS should recover over time; this is a serious weakness of the CS processing idea.

The weight of opinion seems to be converging on the view that extinction is an independent association in its own right (e.g., Estes, 1959; Konorski, 1967; Spear, 1971), where, for example, after establishing a memory of a CS associated with reinforcement, the animal forms another memory of the CS associated with nonreinforcement. This dual-memory notion opens the possibility of associative interference as an explanatory mechanism of SR: The two memories-- one of reinforcement and one of extinction-compete for retrieval. At first the extinction memory wins out, but with the passage of time, the reinforce-

Correspondence should be addressed to L. D. Devenport, Department of Psychology, University of Oklahoma, Norman, OK 73019 (email: ldeven(a)ou.edu). ment memory prevails. However, for the interference model to do anything more than transform a behavioral puzzle into a memory puzzle, it must explain why the memory of extinction is more easily forgotten than that of reinforcement

Kraemer and Spear (1993) accomplished this by suggesting that the two associative memory traces progressively decay, converging on the same threshold for activation, at which point competition for retrieval is about even. This implies that any pair of potentially interfering memories would eventually be retrieved with equal probability. Kraemer and Spear went on to propose that extinction memory is a special case, being less significant or salient than the reinforced memory. In time, therefore, the activation threshold for extinction would rise above that of reinforcement and progressively fail in the competition for retrieval.

Bouton $(1991,1993)$ has taken a somewhat similar view of the matter, although he claims that the weakening of extinction memories stems from a greater context dependence for their retrieval, not a motivational asymmetry. Thus, if time is considered to be a contextual cue, the Bouton model, like Kraemer and Spear's (1993), fits the facts about SR. Models that stress the changing retrievability of competing memories (henceforth "interference models") are superior to, for example, CS processing accounts, because explanations are given for the effects of time.

Beginning with Pavlov (1927), accounts of extinction and its dissipation have continued to be framed in terms of simple associative learning, from which the vast majority of the evidence has been drawn. In fact, it is questionable whether SR and the interference models that explain it extend beyond associative learning. Bouton (e.g., Bouton, 1984; Bouton \& Swartzentruber, 1991), however, proposes that they do. There is good reason to suspect that the suggestion is overspeculative, perhaps of SR, and almost certainly of interference models. 
In their influential review, Sherry and Schacter (1987) distinguished between "procedural" and "declarative" memories. Declarative memory - and here I especially refer to "episodic" memory-preserves variable experiences embedded in time and space. Such memories are acquired rapidly and are resistant to interference. Procedural memories are considered to be records of stable interactions with the environment that are acquired incrementally and that are susceptible to interference at the level of retrieval. Along with response and skill learning, Pavlovian conditioning falls into this category. Procedural memories, unlike declarative memories, are not dependent on the integrity of the hippocampus for their formation (reviewed in Squire, 1992).

The most widely acknowledged instances of declarative memory in animals come from studies of complex spatial learning. Spatial "maps" are representations of important places that can include, among other things, the metric relationships among stable landmarks, the shape or terrain of the landscape, and proprioceptive and vestibular information (reviewed in Gallistel, 1990; see also Collett, Cartwright, \& Smith, 1986). Functionally, maps are valuable because they permit shortcuts, skirting of obstacles, and, of course, keep animals from getting lost when they deviate from fixed routes. Not only does mapping require memory of variable experiences, but it also passes the test of hippocampal dependence (O'Keefe \& Nadel, 1978; Squire, 1992) and is exceptionally resistant to interference, as several studies have shown (Beatty \& Shavalia, 1980; Maki, Brokofsky, \& Berg, 1979; Roberts, 1979). Thus, if SR were to be found in spatial mapping tasks, a new account would be required because interference models no longer pertain. CS processing explanations are difficult to apply outside of their Pavlovian framework, and, as already discussed, have other failings. If SR were to be documented for interferenceresistant tasks, theoretical explanation would be lacking, except for a recent foraging model that, despite being designed for other purposes, would seem to offer a complete account.

With my colleagues (J. A. Devenport \& L. D. Devenport, 1993; L. D. Devenport \& J. A. Devenport, 1994; L. D. Devenport, Hill, Wilson, \& Ogden, 1997), I advanced a foraging theory and model-the temporal weighting rule (TWR) - which, simply expressed, asserts that resource variability is best dealt with by returning to a site that has been yielding well, but only if done quickly, before its quality changes. After an extended absence, the forager's best bet, by TWR, is to select a patch that is usually rich because in the long run this policy will increase efficiency (see the Appendix). What regulates the transition between these seemingly disparate means of valuating patches, one deterministic, the other probabilistic? According to TWR, the answer is time.

Because time is the source of stochastic change, TWR discounts patch outcomes by the length of time, $T$, that has passed between foraging experiences. Accordingly, each experience earns a weight dictated by its recency, $1 / T$. Any such weighted average would be dominated by the last patch outcome, if recent. However, when a forager is interrupted and experiences recede into the past, the initial heavy weighting for recency becomes distributed more and more evenly until, finally, the weighted average of these experiences converges on the unweighted patch average. Choices are now expected to be made on this basis. This is functional because patches vary in their momentary value but, like most biological variables, tend to regress to their mean quality, which differs from patch to patch (see, e.g., McNamara \& Houston, 1987; Pleasants \& Zimmerman, 1979; Rabenold \& Bromer, 1989). Thus, by tying the weighting of experiences directly to time, TWR automatically regulates the transition between tracking recent trends and falling back on more enduring probabilities. A quantitative description of TWR is given in the appendix, with examples of how it is applied to field and laboratory studies.

The output of TWR is a "dynamic average," but from a strictly behavioral perspective, it could be considered to be a model of SR for complex behavior involving spatial and temporal memory. For example, TWR predicts that, despite some bad experiences ("extinction"), animals will eventually return to a patch with a good average (previous reward). Thus, TWR clearly predicts the emergence of SR in complex tasks.

As a model, TWR has several merits. It makes exact predictions about expected preference shifts - that is, the final degree to which initial training "recovers." TWR does not grant special status to extinction. Absence of food at a patch is simply one of many possible experiences that help define the patch's present and long-term quality for an animal. TWR's weighting by recency results in hyperbolic discounting of experiences (see the Appendix). Unlike exponential discounting, hyperbolic discounting proceeds at different rates at different times, discounting steeply at first and then more slowly. With temporal discounting, a second experience will always discount at a faster rate than the first until, for all practical purposes, it carries the same weight. Thus, TWR automatically performs the Kraemer and Spear (1993) "convergence" process, and, in general, solves the problem of how a second experience (like extinction) does not continue to prevail. However, TWR lacks the merit of empirical support. The tests to which it has been submitted (J. A. Devenport \& L. D. Devenport, 1993; L. D. Devenport \& J. A. Devenport, 1994; L. D. Devenport et al., 1997) did not rule out the possibility of associative learning and interference. Accordingly, I devised a study to determine whether SR does, in fact, emerge from a spatial mapping task, and if so, whether TWR can offer a credible account.

\section{EXPERIMENT 1}

I designed a spatial mapping problem in which rats first learned that one patch was baited and the other was 
empty. In the second stage of training, conditions were reversed, so that the previously baited patch was empty and the other was baited. Thus, the animals were given a reward and extinction experience at each patch. TWR predicts that, as a natural consequence of dynamic averaging, SR will mount with the passage of time. TWR also predicts that when the averaging processes is complete, the proportion of animals choosing a particular patch will be a function of the respective patch averages. To test this prediction, I made a pair of patches of unequal average value for one set of animals, but of equal average value for a second set, and tested their patch preferences at various delays. Since an unweighted average (emerging after long delays) cannot be computed without a memory of both stages of training, this manipulation of patch quality also assessed the retrievability of patch memories acquired during both stages of training.

Unlike natural settings and the present experiment, laboratory studies of SR commonly provide no alternatives. The only "choice" given an animal is whether to respond or not. Consequently, it is hard to specify when SR will emerge and with what strength. Even a slight possibility that food might once again be available could trigger SR in a highly deprived animal, but SR could take longer for less desperate subjects. In part, this nochoice procedural convention may have led to the longstanding belief that reinforcement is in some way stronger or more retrievable than nonreinforcement. Survival depends on trying to obtain food, even when the odds are unfavorable. Choice experiments are not so sensitive to these complications because animals can usually be counted on to choose the better patch, irrespective of subject variables. Thus, because the natural environment always contains options and because choice paradigms admit of more exact predictions (see the appendix for further documentation), I used them in the present experiments.

Although spatial mapping problems are quite resistant to interference, this is probably true only up to a point (see, e.g., Roberts, 1979). Accordingly, I gave animals only two patches with two outcomes at each, a far lighter memory load than found in most mapping studies. Finally, to remove any lingering doubts about the intrusion of forgetting, I tested the animals' spatial memory for evidence of interference, as will be explained.

\section{Method}

\section{Subjects}

Sprague-Dawley male rats $(N=62)$ were maintained in individual hanging steel cages with constant access to water and rodent chow (Purina, St. Louis), except as noted. Fluorescent lighting came on at 7 a.m. and went off at 7 p.m., and the animals were trained and tested during the light phase of their cycle. Before training, they were brought to $80 \%$ of their free-feeding body weight (which ranged from 320 to $400 \mathrm{~g}$ ) by reducing food allotments and at the same time introducing a few of the unhulled sunflower seeds (black oil type) to be used in training.

\section{Apparatus}

Animals foraged for buried sunflower seeds in one of two large sandboxes built of wood, measuring $1.2 \times 2.8 \times .28 \mathrm{~m}$ and elevated $18 \mathrm{~cm}$ above the floor. The apparatus was loosely designed after Vander Wall (1991). Covers constructed of hardware cloth supported by a rectangular frame were attached to the boxes, which were filled with about $800 \mathrm{lb}$ of sterilized sand to a depth of about $12 \mathrm{~cm}$. Natural items (a short tree limb, a small stump, and two rocks) were partially buried in the sand for use as spatial references. These objects were replaced or rearranged periodically in each of the foraging boxes. The four sides of the boxes were marked on the exterior with a linear scale, providing 2-D coordinates of items in the box. Using this grid, a map of the contents of the box was constructed, permitting readjustment of embedded objects in case they were inadvertently moved. A single water bottle was attached to the outside of the box with its spout protruding $2 \mathrm{~cm}$ into the box.

Four pretraining boxes of the same proportions as the foraging boxes, but only one quarter of the area, were constructed in the same way and filled to the same depth with sand. Two items, a small $\log$ and a rock, were partially buried in the sand in each pretraining box. The sand in the pretraining and foraging boxes was periodically replaced from a fresh supply kept in reserve.

\section{Procedure}

Pretraining. Pretraining was carried out in the small boxes where the rats practiced digging for unhulled sunflower seeds. Although rats dig spontaneously, it was necessary to teach them to dig especially deep for the buried patches they would eventually have to excavate. Deeply buried patches in desiccated sand suppress olfactory cues and tactile cues (L. D. Devenport, Humphries, \& J. A. Devenport, in press; Vander Wall, 1991) and, most importantly, encourage vigorous digging that would be unmistakable to observers during tests.

At first, two continuous columns of about 100 seeds each were prepared by filling a long glass test tube with seeds, inverting it and thrusting it to the base of the training box and then slowly removing it, resulting in a more or less continuous column of seeds for the rats to extract. On the 1 st day of pretraining, two patches were prepared, one that remained in the same position on subsequent pretraining trials and one that moved. The patches varied in quality, one usually containing $80-100$ seeds and the other $20-40$ seeds. They were prepared by removing sand with a trowel, depositing the seeds, and simply covering them with sand. The most superficial seeds in the patches were buried shallowly and the position of each patch was marked on the surface with three seeds. These marker seeds usually induced prompt digging, which revealed the patch below. The animals were allowed to forage until they had appeared to deplete the patches or had stopped digging. On the 3 rd and 4 th days, the patches were buried progressively deeper until they were at least $4 \mathrm{~cm}$ beneath the surface of the sand. The patch in the consistent position was unmarked on these subsequent days and had to be located from memory. The patch in the varying position was marked with three surface seeds as before. Using both varying and stable patches left the animals with no consistent expectations that might be transferred to formal training.

Training. Animals received formal training in one of the large sandboxes within the next 2 or 3 days. The rats were allowed to explore their assigned foraging box for at least an hour the day before formal training, with no seeds present in the box. On the next day, two patches of seeds were prepared. Patch sites differed among the animals and were positioned randomly throughout the box, except that they were required to be at least $1 \mathrm{~m}$ apart and well away from an object that might serve as a beacon for localization. To ensure accurate positioning, a set of Plexiglas squares and rectangles of various sizes was used. A corner of one of these "patch locators" 
was held firmly in a predetermined corner of the box, and the point diagonal to this corner identified the patch site. A second locator, chosen from a reserve of varying sizes, was used in another corner of the box to position the second patch site.

Patches were prepared using a trowel to remove about $8 \mathrm{~cm}$ of sand and then thrusting a section of PVC pipe ( $4 \mathrm{~cm}$ in diameter) to the base of the box. The pipe was filled with sand to a first mark, and then with about one third of a precounted number of seeds, a small layer of sand, another one third of seeds, more sand, and so forth, to a final mark, after which sand alone was deposited, bringing it level with that of the rest of the box. Because differing volumes of seeds were used for some groups, we varied the depth of the patches to yield an approximately constant seed density. Thus, although richer patches extended more deeply, all patches required the rats to remove at least $4 \mathrm{~cm}$ of sand before exposing seeds, and all patches were $4 \mathrm{~cm}$ in diameter. After the sand was raked and smoothed to a uniform appearance throughout the box, the animal was introduced from varying locations, the lid closed, and two observers recorded foraging activities.

During the first foraging session, two patches, $\mathrm{A}$ and $\mathrm{B}$, were available. Patch $B$ was marked with three seeds, but was otherwise empty. After the animal had ceased to dig at Patch B for 1 continuous min, the seed-filled patch (Patch $\mathrm{A}$ ) was marked by dropping three sunflower seeds directly on it through the hardware cloth cover (the exact patch position was marked on the exterior of the cover with chalk). Because each animal was assigned different patch positions, chalk odor at multiple places would have been an unreliable cue (indeed, no animal ever discovered a filled patch until it was marked with seeds, as described). After finding the markers at Patch $\mathrm{A}$, the animal was allowed to dig and extract seeds for a predetermined duration. Only digging and eating behavior was timed. Extraneous activities away from the patch such as drinking or exploring were not included. When the allotted foraging time at the second patch had elapsed, the animal was removed from the box and placed in a polycarbonate holding cage with water, but no food. The patches were sifted for remaining seeds, from which we obtained an accurate count of the number taken. Seed hulls and other debris were also removed by sifting, and the sand was redistributed about the box using garden tools.

The second foraging session began $3 \mathrm{~h}$ later, with both Patches $A$ and $B$ marked with surface seeds. Patch A was empty, but Patch $B$ was filled with the assigned number of seeds. The animals invariably dug where they had previously found seeds (Patch A) and were allowed to do so until they spontaneously gave up and tried Patch B. As before, animals were given an allotted time to extract seeds from the baited patch (B).

One set of animals $(N=31)$ was given patches of greatly differing average quality $(A>B)$. For them, Patch $A$ was filled with 180 seeds on the first foraging trial, and they were allowed $6 \mathrm{~min}$ to dig, extract, and eat. On the second trial, Patch B contained 30 seeds and the rats were allowed only $1 \mathrm{~min}$, after they had given up on Patch A, to extract and eat. The aim was to allow the animals sufficient time to assess the patches, but not enough time to deplete them by more than $50 \%$. Because, as the results will show, extraction efficiency differed between patches in the A $>B$ group, the time limits were relaxed in a few cases to ensure that patches were not over- or underexploited. The study therefore did not permit assignment of an exact quantitative value to a patch, but it ensured that the qualitative $(\mathrm{A}>\mathrm{B})$ difference was met by a wide margin. A second set of animals $(N=31)$ was given patches of equal average quality $(A=B)$. During their first foraging trial, Patch $A$ was filled with 100 seeds and Patch B was empty; during the second session, Patch B was filled with 100 seeds and Patch A was empty. All procedures were the same as described for the $\mathrm{A}>\mathrm{B}$ condition, except the animals were allowed to collect seeds from each patch for $5 \mathrm{~min}$. No temporal adjustments were required for these groups because foraging efficiency was the same for both patches.
Test. Because of its strong weighting for recency, TWR predicts that the last patch to yield will be preferred for a limited time, irrespective of its size. However, as the relative recencies of the first and last foraging trials converge, the dynamic average of patch quality will barely differ from the unweighted average. Consequently, I expected a shift toward indifference on the latest tests for the $\mathrm{A}=\mathrm{B}$ condition. For the $\mathrm{A}>\mathrm{B}$ condition, I expected an earlier shift, ending with a decided preference for Patch A because of its much better average quality.

To track these expected shifts, independent groups of animals were given patch choice tests at various intervals following the last training session. I used TWR (see equations in the appendix) to identify delays that would capture the expected dynamics, although projections were necessarily approximate owing to a design that was more concerned with memory than with exact determination of temporal dynamics. The six train-test delays used$0.5,2.5,6,12,24$, and $48 \mathrm{~h}$-were expected to bracket the preference shifts. The number of animals assigned to each of the 12 groups is given in the caption of Figure 1. During test, the patch sites were empty, unmarked, and the box was sifted of debris and raked smoothly. In order to help rule out travel time or sheer convenience as choice factors, the rats were introduced into the box from positions approximately intermediate between patch sites. The rats were thus required to estimate the relative worth and location of the patches from memory. As commonly accepted, their first choice was regarded as a direct reflection of patch preference. However, the number of return visits by the animals to the firstchosen patch before shifting to the alternate patch was also recorded, with the expectation that these data would verify that the first choices were not capricious.

Two observers seated on opposite sides of the foraging box recorded which patch was chosen first and the time spent digging there. Finding no seeds, the rats usually departed and groomed, drank from the water spout, or ran once or twice around the perimeter of the box. The duration of this nondigging interval was recorded, along with the patch chosen after foraging resumed, the duration of that dig, and so forth, until the alternate patch was selected. Thus, rather than removing the animal after it made a patch choice, time was allowed to determine whether the animals could locate and dig up the nonpreferred patch.

Judgment of the accuracy of patch choices by observers was aided by the linear scale on the sides of the boxes, the maps of the box and patch sites, and especially the chalk mark made on the hardware cloth cover, as described before. The accuracy of patch localization was of course limited to the visual resolution of the observers using these aids. Observer accuracy was determined in preliminary tests. The patches were $4 \mathrm{~cm}$ in diameter, and a dig anywhere within this area plus an additional $1.3 \mathrm{~cm}$ (observer error) was recorded as an exact localization. Any digging beyond this limit, regardless of distance, was considered "off-patch." Offpatch digs were common after the animals had found both patches to be empty. These were not counted as errors, but were taken to mean that the rats had given up on both patches. If they refused to dig at the true patches for 5 consecutive min, testing was ended.

Statistical analysis. Descriptive statistics were calculated for train and test measurements. The between-and within-groups data were analyzed for possible differences using two-tailed $t$ tests. I used logistic regression analysis for the patch choice data collected at test, with patch condition $(\mathrm{A}>\mathrm{B}, \mathrm{A}=\mathrm{B})$ and test delay (the six test intervals) as predictor variables. Individual, not aggregate, data were used for the regression analysis. Logistic regression is expressly designed for binary dependent variables, for which continuous or categorical variables are used as predictors, and is therefore the most appropriate analysis. Probabilities of accurate patch localization were calculated by dividing patch area by box area, minus area occupied by the embedded objects (see L. D. Devenport et al., in press; Vander Wall, 1991). 


\section{Results and Discussion}

\section{Training}

As can be seen in Table 1 , the $A=B$ groups collected an almost equal number of seeds at each patch, but the A $>$ B groups experienced about a 3:1 ratio. Table 1 also gives the seed capture rates, which were very similar for the $A=B$ patches, but somewhat uneven for the $A>B$ patches. Although open to interpretation, at the large patches, where extended digging was allowed, heaps of excavated sand periodically collapsed and had to be removed again. This did not happen as frequently at the small patches, possibly allowing for more rapid extraction. In any case, a wide difference in the quality of the $\mathrm{A}>\mathrm{B}$ patches was established, but assignment of exact patch values was precluded because the relative importance of amount extracted and extraction rate could not be assessed after the fact.

\section{Test}

Dig times for test are given in Table 1, showing that our pretraining procedures produced long and vigorous digging before the animals gave up, allowing observers to fully concur about the order of patch choices and the spatial accuracy of the digging at both the favored and the alternative patches.

Figure 1 illustrates the shifts in patch preference (first choices) over time and provides unequivocal evidence of SR. Three features of these data bear mention. First, irrespective of the patch quality during training for each group ( $\mathrm{A}=\mathrm{B}, \mathrm{A}>\mathrm{B})$, all animals chose the patch $(\mathrm{B})$ that yielded seeds in the last stage for up to at least $2.5 \mathrm{~h}$ posttraining. Second, after that interval, the $A>B$ animals' preference for Patch B declined rapidly, until at both 24 and $48 \mathrm{~h}$ posttraining, every member of the $A>$ $B$ group selected Patch $A$ as its first choice at test. Third, the choices of the $A=B$ groups also shifted, but their final preferences settled on indifference. The choice dynamics in Figure 1 are clearly structured and are just as predicted by TWR, although the conclusion would have been strengthened if another test delay had been included to ensure that the $A=B$ group remained indiffer-

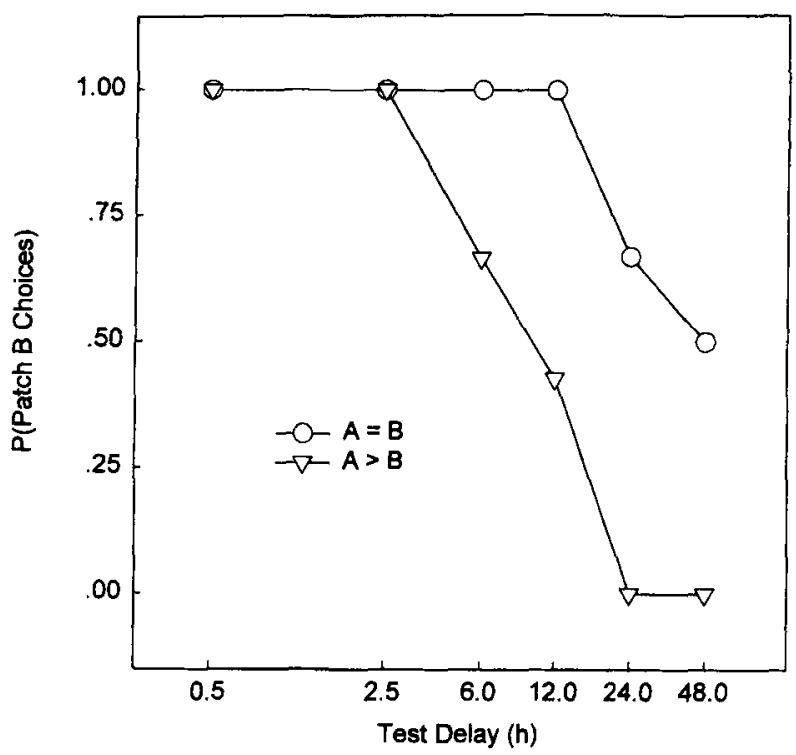

Figure 1. Proportion of animals in each patch condition $(A>B$ and $A=B$ ) choosing Patch $B$ across train-test delays. Note that a proportion of .5 means that half the animals in that group chose Patch $A$ and half chose Patch $B$; a proportion of .0 means that all animals in that group chose Patch $A$. For the $A=B$ conditions, the $n s$ for the six respective test delays shown are $5,2,4,6,6$, and 8; those for the $A>$ $B$ condition are $5,2,6,7,5$, and 6 .

ent, as we have previously shown using different procedures (L. D. Devenport \& J. A. Devenport, 1994; L. D. Devenport et al., 1997).

Logistic regression analysis confirmed that the twovariable model (test delay and patch condition) was strongly predictive of individual choices $\left[\chi^{2}(2, N=62)\right.$ $=40.4, p<.00001]$. To determine the contribution of patch condition, I removed this variable from the model and found that accuracy of the one-variable regression model was significantly reduced compared with that of the two-variable model $\left[\chi^{2}(1, N=62)=17.0, p<.00001\right]$. Thus, patch condition contributed roughly $42 \%$ to the predictive power of the regression model, whereas time, of course, accounted for the rest. Table 1 shows a signif-

Table 1

Patch Conditions and Foraging Activities of Rats During Training and Test in Experiment 1

\begin{tabular}{|c|c|c|c|c|c|c|c|c|c|c|c|}
\hline \multirow{3}{*}{$\begin{array}{l}\text { Patch Condition } \\
\text { (Groups) }\end{array}$} & \multicolumn{5}{|c|}{ Training } & \multicolumn{6}{|c|}{ Test } \\
\hline & \multirow{2}{*}{$\begin{array}{l}\text { Seeds } \\
\text { Given }\end{array}$} & \multicolumn{2}{|c|}{$\begin{array}{l}\text { Seeds } \\
\text { Eaten }\end{array}$} & \multicolumn{2}{|c|}{$\begin{array}{c}\text { Capture } \\
\text { Rate }\end{array}$} & \multicolumn{2}{|c|}{$\begin{array}{l}\text { Test Dig } \\
\text { Time }\end{array}$} & \multicolumn{2}{|c|}{$\begin{array}{c}\text { Digs to } \\
\text { Shift }\end{array}$} & \multicolumn{2}{|c|}{$\begin{array}{l}\text { Time to } \\
\text { Second Dig }\end{array}$} \\
\hline & & $M$ & $\overline{S E M}$ & $M$ & $S E M$ & $M$ & $\overline{S E M}$ & $M$ & SEM & $\bar{M}$ & $\overline{S E M}$ \\
\hline$A=B$ & & & & & & & & $1.78 \dagger$ & $\pm 0.172 \dagger$ & 34.5 & \pm 6.47 \\
\hline Patch A & 103 & 50.0 & 2.30 & 0.152 & 0.006 & 22.4 & \pm 9.7 & & & & \\
\hline Patch B & 103 & 47.7 & 1.40 & 0.154 & 0.005 & 19.9 & \pm 3.3 & & & & \\
\hline$A>B$ & & & & & & & & $1.78 \dagger$ & $\pm 0.152 \dagger$ & 36.59 & \pm 9.33 \\
\hline Patch A & 183 & 58.4 & 1.82 & 0.156 & 0.008 & 25.9 & \pm 9.2 & & & & \\
\hline Patch B & 33 & 15.4 & 0.08 & $0.169 *$ & $0.016^{*}$ & 23.0 & \pm 3.3 & & & & \\
\hline
\end{tabular}

Note-Seeds given $=$ total number of seeds, including markers, when patch was filled. Capture rate $=$ seeds extracted and eaten per second. Test dig time $=$ total dig time at each patch during test. Digs to shift $=$ return visits to first patch choice before switch to alternate patch at test. Time to second dig = seconds between first and second patch choices at test. ${ }^{*} p<.05$ compared with alternative patch, correlated $t$ test, two-tailed. ${ }^{t} p<.0001, t$ test, two-tailed, testing the hypothesis that return visits $=0$. 
icant tendency for animals to depart briefly after finding their first choice empty, but then to return at least once before switching to the alternative. Thus, the initial preferences shown in Figure 1 were matched by the reluctance of the animals to give up on this choice. The rats in both patch conditions displayed extremely accurate spatial localization of their first choice, with all meeting the criterion of precision.

A critical component of testing was to determine whether the animals could locate and dig up the alternate patch, showing that they remembered where it was and that it had once contained seeds. As with the first choice, every animal located and dug at the alternate patch with the same accuracy (i.e., precise without exception, $p<$ .002 for each animal), indicating that the rats knew the exact location of both sites from among the thousands possible, and that digging might uncover seeds. The patch choice dynamics also attest to seemingly complete knowledge of both stages of training. How else, after SR was complete $24-48 \mathrm{~h}$ posttraining, could $\mathrm{A}=\mathrm{B}$ animals know that the patches were of equivalent value, and $\mathrm{A}>\mathrm{B}$ animals know that Patch $\mathrm{A}$ was best?

\section{EXPERIMENT 2}

Although the results of Experiment 1 seemed quite decisive, I designed this experiment to remove as much uncertainty as possible about our success in generating SR in the absence of interference. Skeptics might mount a context-cuing interpretation of Experiment 1 as follows: SR springs from the differing retrievability of competing memories excited by contextual cues present at the time of test. Might not the rats' precise localization of the second choice at test be attributable to the extinction experience at the first-chosen patch, thereby switching memory retrieval to the stage of training in which such conditions once prevailed (Brooks \& Bouton, 1993; but see Robbins, 1990)? While possible, this argument fails to account for how animals in each condition knew the relative value of their patches. For that, they needed access to both stages of training.

Nevertheless, I determined whether spatial accuracy for the alternate patch would be compromised by eliminating the extinction experience at the first-chosen patch. I did this by preventing the animals from sampling their preferred patch.

\section{Method}

\section{Animals and Procedure}

Animals $(N=9)$ were of the same body weight and sex as in Experiment 1 . They were pretrained as previously described, but assigned to the $\mathrm{A}>\mathrm{B}$ condition only, using the same training procedures as before. However, during the 1 -h exploratory opportunity the day before formal training, they were habituated to a clear Plexiglas cylinder $(29.5 \mathrm{~cm}$ in diameter $)$ inserted in a random location in the foraging box. When thrust to the floor of the sandbox, the cylinder was just high enough to contact the hardware cloth cover of the foraging box, so the rats could not climb in. The cylinder was not present during the two formal training trials.
I tested roughly half the rats at $2.5 \mathrm{~h}$ posttraining $(n=4)$ and the rest at $24 \mathrm{~h}$ posttraining $(n=5)$, delays at which preferences were decidedly in favor of Patch B and Patch A, respectively, in Experiment 1 (see Figure 1). During test, neither patch was marked by superficial seeds, nor were they filled. For the 2.5-h train-test group, access to Patch B was prevented by the cylinder, and for the 24-h train-test delay animals, Patch A was blocked. Thus, the rats could inspect the area around the blocked patch and see the patch site through the cylinder, but could not access it. Being thwarted from exploiting their preferred patches, I expected that the animals would turn to their less preferred patches and dig with the same accuracy as the subjects in Experiment 1, showing that the precise memory displayed in Experiment 1 did not depend on inadvertent cuing that might have facilitated retrieval of otherwise unavailable memories.

\section{Results and Discussion}

During training, the animals obtained $55.8 \pm 5.4$ seeds from Patch A and $14.4 \pm 1.2$ seeds from Patch B, closely resembling the respective amounts taken by the $\mathrm{A}>\mathrm{B}$ animals in Experiment 1 (compare with results in Table 1). Similarly, the rats in this experiment had higher extraction rates when foraging at Patch $B$ than at $\mathrm{A}(.232$ $\pm 0.038 \mathrm{seeds} / \mathrm{sec}$ and $0.197 \pm 0.016 \mathrm{seeds} / \mathrm{sec}$, respectively). However, the difference was not significant in this experiment, probably owing to the smaller $N$.

At test, every rat dug at the exact location of the unblocked patch ( $p<.002$ for each rat) and did so vigorously and unmistakably for an average of $16.8 \pm 2.79 \mathrm{sec}$. Thus, without any known cuing, the results show that the animals remembered all the important details of their training experiences. Habituating the animals to the cylinder prior to training helped rule out the possibility that the introduction of this patch-blocking device during test might have influenced memory retrieval in some way. Indeed, the cylinder was, if anything, a cue that the box contained no seeds, because it had been associated with an empty foraging box. Thus, in spite of the cylinder, the animals performed perfectly.

\section{GENERAL DISCUSSION}

SR made a robust appearance in this spatial mapping task, whose known resistance to interference was reaffirmed. Regardless of when they were tested, the rats in Experiment 1 pinpointed the location of both patches against very difficult odds, and did so without the aid of inadvertent retrieval cues, as shown by Experiment 2 . Added to this, patch quality was found to be a highly significant predictor of choice in Experiment 1, accounting entirely for the divergence between groups at the more delayed tests when SR was complete, further indicating that the rats remembered the essential components of all their training experiences.

Since no evidence of forgetting was expected, nor any found, the SR produced by this spatial mapping task cannot be attributed to interference. Accordingly, Bouton's (1994) extension of interference models to foraging was premature. I know of no account, except that of TWR, that can explain why an animal would revert to a behav- 
ior so thoroughly replaced by another during training. The rats in the present study did exactly that, and the reversion was by no means unstructured. Animals for whom the two patches were of equivalent worth $(A=B)$ divided their choices evenly between them at the late test, and all those for whom Patch A was better chose it exclusively at the late tests, but both groups chose Patch B at early tests. I conclude that the animals judged patch value at every test delay by a dynamic averaging process, just as TWR predicts.

From a biological perspective, dynamic averaging (and its expression as SR) helps animals to cope with environmental variability by exploiting the nearly deterministic patch conditions that follow a recent experience, but also the probabilistic conditions that prevail when time passes and patches regress to their mean quality. Another behavioral phenomenon, the discounting of delayed rewards, has also been modeled most successfully using temporal (hyperbolic) discounting of expected outcomes (see, e.g., Kirby \& Herrnstein, 1995; Mazur, 1994), and the adaptive value of doing so is most commonly ascribed to environmental variability - the longer the wait, the less likely that expectations will be fulfilled. Thus the model of "past discounting," as TWR might be loosely regarded, bears a kind of symmetry with "future discounting," suggesting that animals may use time-based solutions to deal with prospects that become increasingly uncertain with time.

TWR accomplishes much with very little. The model is built around two well-established facts, timing and averaging. Inhibition, excitation, associations, activation thresholds, differential context dependence, forgetting, and the notion of extinction itself do not clutter the model. I would hasten to add, however, that TWR is not designed for, nor is it equipped to explain, the countless phenomena for which these constructs are indispensable. TWR almost certainly applies to a behavioral domain separate from associative learning, as discussed from a theoretical perspective earlier, and which this study further documents.

The theory behind TWR was formulated a priori as an animal's best guide to patch assessment in the face of environmental variability. Thus, it explained in advance why SR should evolve, passing the critical test of biological utility before the theory was put to test. Our field experiments with domestic dogs (J. A. Devenport \& L. D. Devenport, 1993) and with wild populations of golden-mantled ground squirrels (Spermophilus lateralis) and least chipmunks (Tamias minimus) (L. D. Devenport \& J. A. Devenport, 1994) have shown that the output of TWR - dynamic averaging-is used spontaneously in natural settings. Thus TWR is not confined to the laboratory. At least as far as SR is concerned, TWR and its theoretical foundation is probably best regarded as a predictive model, a potential mechanism, and an explanation for complex information processing.

TWR can make a host of other predictions about SR besides those already discussed; these need to be tested.
For instance, if an animal is trained with two patches, one that undergoes extinction and one that continues to yield during both stages of training, TWR predicts no appreciable SR because the average quality of the stable patch will be greater across all possible train-test delays. Interference models predict that extinction memories are eventually forgotten, so animals that obeyed such models would be expected to display SR in the form of indifference, regarding the patches as equals.

TWR also predicts that the appearance of SR will not be subject to a fixed delay. Instead, the delay should be an orderly function of the temporal separation of patch experiences (or stages of training): The further separated, the longer the last experience should exert its effect, thereby delaying the emergence of SR. Even if the two training stages are contiguous, the length of time each successive patch condition or contingency prevails should determine how long it will take SR to appear. By TWR, recency is not fixed, but is relative to rate of change, in keeping with the scalar property of animal timing (Gibbon, 1977; Gibbon, Baldock, Locurto, Gold, \& Terrace, 1977). If this prediction were not borne out, the adaptive value of dynamic averaging and the predictive power of TWR would be badly compromised because the rate of change of a resource sets the length of time over which conditions can be expected to remain unchanged. Information based on last experiences at fastchanging patches should become obsolete sooner than similar information from slowly changing patches. Interference theorists of SR have not addressed this issue, nor have they justified the test delays used in their studies. Unless the models are given quantitative expression, I do not see how they could.

TWR also predicts that neither extinction nor counterconditioning is necessary for the appearance of SR. Instead, simple shifts in the relative richness of patches or laboratory alternatives should serve to engender SR. Mazur $(1995,1996)$ has already tested and confirmed this prediction using concurrent schedules of reinforcement that varied in richness. In his studies, pigeons exploited both schedules during each session, but they directed more responses toward the target associated with the richer schedule until the relative richness of the schedules was equalized, whereupon responses were evenly distributed. After a day's delay, however, the birds temporarily reverted to their original preference for the once-rich patch until discovering that conditions were unchanged.

TWR also predicts that if the order of training were reversed, SR would still occur. Suppose animals first learned that the concurrent schedules were of equal richness, and then that one was better. After a delay, the newly acquired preference for the richer patch should diminish considerably as the weighting accorded the last stage of training diminished and that of the first stage increased. Mazur (1996) reported exactly this result.

The present study has shown for the first time that a simulated foraging task, requiring retention of spatial, 
temporal, and patch quality memories, displays all the attributes of SR. This is significant for several reasons, one of the most important being that interference among memories is not a necessary condition for SR in this domain. However, nothing in this study weakens the possibility that many of the laboratory settings and procedures used to generate SR do so through forgetting, as claimed by advocates of interference models. I conclude that SR can spring from very different mechanisms: interference for simple associative memories, perhaps along with factual memories devoid of importance (e.g., word lists) and dynamic averaging for more complex behavior.

The diversity of paradigms used in behavioral research makes it difficult to know exactly where to draw the line between simple and complex learning and memory. Many tasks, arguably, contain components of associative learning as well as more elaborate cognitive processes. Deciding between TWR-like effects and those of interference is also impeded by the slim evidence on which claims of forgetting are so frequently based. Simply failing to respond or failing to respond as expected is the commonly accepted standard. Indeed, had I not selected a task known to be resistant to interference and designed the study to positively refute forgetting hypotheses, an interference model could probably be molded to account for the results, although it would struggle to explain the divergent patch preferences of the two groups.

In summary, interference models of SR have enjoyed considerable success in explaining the spontaneous recovery of simple associatively learned responses. In spite of speculation to the contrary (Bouton, 1994; Bouton \& Swartzentruber, 1991), the models do not stand up to more complex learning. Nor should they. The vast majority of vertebrates repeatedly travel to and from their burrows, dens, nests, or roosts to spatially scattered resources. It is difficult to imagine anything more deserving of unfailing memory than the location and behavior of these resources. A stock of relatively permanent information, along with rules for how to use it, appears necessary for survival in the wild (see J. A. Devenport \& L. D. Devenport, in press).

\section{REFERENCES}

Beatty, W. W., \& Shavalia, D. A. (1980). Rat spatial memory: Resistance to retroactive interference at long retention intervals. Animal Learning \& Behavior, 8, 550-552.

Bouton, M. E. (1991). Context and retrieval in extinction and in other examples of interference in simple associative learning. In L. Dachowski \& C. F. Flaherty (Eds.), Current topics in animal learning: Brain, emotion, and cognition (pp. 25-53). Hillsdale, NJ: Erlbaum.

Bouton, M. E. (1993). Context, time and memory retrieval in the interference paradigms of Pavlovian learning. Psychological Bulletin, 114, 80-99.

Bouton, M. E. (1994). Conditioning, remembering, and forgetting. Journal of Experimental Psychology: Animal Behavior Processes, 20, 219-231.

Bouton, M. E., \& Swartzentruber, D. (1991). Sources of relapse after extinction in Pavlovian and instrumental learning. Clinical Psychology Review, 11, 123-140.

Brooks, D. C., \& Bouton, M. E. (1993). A retrieval cue for extinction attenuates spontaneous recovery. Journal of Experimental Psychology: Animal Behavior Processes, 19, 77-89.

Brunner, D., Gibbon, J., \& Fairhurst, S. (1994). Choice between variable and fixed delays with different rewards. Journal of Experimental Psychology: Animal Behavior Processes, 20, 331-346.

Collett, T. S., CARTwright, B. A., \& SMith, B. A. (1986). Landmark learning and visuo-spatial memories in gerbils. Journal of Comparative Physiology A, 158, 835-851.

Devenport, J. A., \& Devenport, L. D. (1993). Time-dependent decisions in dogs (Canis familiaris). Journal of Comparative Psychology, 107, 169-173.

DevenPort, J. A., \& Devenport, L. D. (in press). Squirrel foraging behaviour. In G. Greenberg \& M. H. Haraway (Eds.), Handbook of comparative psychology. New York: Garland.

DEVENPORT, L. D., \& DevenPorT, J. A. (1994). Time-dependent averaging of foraging information in least chipmunks and golden-mantled ground squirrels. Animal Behaviour, 47, 787-802.

Devenport, L. D., Hill, T., Wilson, M. J., \& OGden, E. (1997). Tracking and averaging in a variable environment: A transition rule. Journal of Experimental Psychology: Animal Behavior Processes, 23, 450-460.

Devenport, L. D., Humphries, T. W., \& Devenport, J. A. (in press). Future value and patch choice in least chipmunks. Animal Behaviour.

Estes, W. K. (1959). Toward a statistical theory of learning. Psychological Review, 57, 94-107.

Gallistel, C. R. (1990). The organization of learning. Cambridge, MA: MIT Press, Bradford Books.

GiBBon, J. (1977). Scalar expectancy theory and Weber's law in animal timing. Psychological Review, 84, 279-325.

Gibbon, J., Baldock, M. D., Locurto, C., Gold, L., \& Terrace, H. S. (1977). Trial and intertrial duration in autoshaping. Journal of Experimental Psychology: Animal Behavior Processes, 3, 264-284.

Green, L., Fristoe, N., \& Myerson, J. (1994). Temporal discounting and preference reversals in choice between delayed outcomes. Psychonomic Bulletin \& Review, 1, 383-389.

Kirby, K. N., \& Herrnstein, R. J. (1995). Preference reversals due to myopic discounting of delayed reward. Psychological Science, 2, 8389.

KONORSKI, J. (1967). Integrative activity of the brain. Chicago: University of Chicago Press.

Kraemer, P., \& Spear, N. E. (1993). Retrieval processes and conditioning. In T. R. Zentall (Ed.), Animal cognition (pp. 87-107). Hillsdale, NJ: Erlbaum.

MAKI, W. S., BROKOFSKY, S., \& BERG, B. (1979). Spatial memory in rats: Resistance to retroactive interference. Animal Learning \& Behavior, 7, 25-30.

MAZUR, J. E. (1994). Effects of intertrial reinforcers on self-control choice. Journal of the Experimental Analysis of Behavior, 61, 83-96.

MAZUR, J. E. (1995). Development of preference and spontaneous recovery in choice behavior with concurrent variable-interval schedules. Animal Learning \& Behavior, 23, 93-103.

MAZUR, J. E. (1996). Past experience, recency, and spontaneous recovery in choice behavior. Animal Learning \& Behavior, 24, 1-10.

McNamara, J. M., \& Houston, A. I. (1987). Memory and the efficient use of information. Journal of Theoretical Biology, 125, 385-395.

O'KeEFE, J., \& NADEL, L. (1978). The hippocampus as a cognitive map. Oxford: Oxford University Press, Clarendon Press.

Pavlov, I. P. (1927). Conditioned reflexes. (G. V. Anrep, Trans.). London: Oxford University Press.

Pleasants, J. M., \& Zimmerman, M. (1979). Patchiness in the dispersion of nectar resources: Evidence for hot and cold spots. Oecologia, 41, 283-288.

Rabenold, K. N., \& Bromer, W. R. (1989). Plant communities as animal habitats: Effects of primary resources on the distribution and 
abundance of animals. In W. G. Abrahamson (Ed.), Plant-animal interactions (pp. 291-353). New York: McGraw-Hill.

REsCoRla, R. A., \& WAGNeR, A. R. (1972). A theory of Pavlovian conditioning: Variations in the effectiveness of reinforcement and nonreinforcement. In A. H. Black \& W. F. Prokasky (Eds.), Classical conditioning II: Current research and theory (pp. 64-99). New York: Appleton-Century-Crofts

RobBins, S. J. (1990). Mechanisms underlying spontaneous recovery in autoshaping. Journal of Experimental Psychology: Animal Behavior Processes, 16, 235-249.

RoBerTs, W. A. (1979). Spatial memory in the rat on a hierarchical maze. Learning \& Motivation, 10, 117-140.

SHERRY, D. F., \& SCHACTER, D. L. (1987). The evolution of multiple memory systems. Psychological Review, 94, 439-454.

SPEAR, N. E. (1971). Forgetting as retrieval failure. In W. K. Honig \& P. H. R. James (Eds.), Animal memory (pp. 45-109). New York: Academic Press.

SQUIRE, L. R. (1992). Memory and the hippocampus: A synthesis from findings with rats, monkeys, and humans. Psychological Review, 99, 195-231.

VANDER WALL, S. B. (1991). Mechanisms of cache recovery by yellow pine chipmunks. Animal Behaviour, 41, 851-863.

\section{APPENDIX Quantifying and Applying TWR}

The temporal weighting rule (TWR) was formulated a priori as a model of patch assessment in a variable environment (J. A. Devenport \& L. D. Devenport, 1993; L. D. Devenport \& J. A. Devenport, 1994), and its output is a dynamic, ever-changing average. This dynamic average is the result of weighting the quality of a patch experience, $Q$, by its recency $1 / T$. Recency is the reciprocal of the stretch of time between a patch experience and the moment an animal prepares to resume foraging. For convenience, in the examples given, timing starts from the end of a foraging bout (or, for laboratory studies, the end of a particular stage of training). Thus, in its simplest form, the temporally weighted value, $v w$, of a particular patch is

$$
v w=(Q * 1 / T) \div 1 / T \text {. }
$$

where the product of the estimated patch quality and its recency is divided by its recency. Of course, an average of a single event, weighted or not, will always $=Q$. To elaborate on Equation $\mathrm{Al}$ for the interesting cases in which the number of patch visits exceeds 1 , the $n$ set of unique patch quality experiences, $Q_{i}$, is summed after each experience is weighted by its respective recency and then divided by the sum of the recencies to obtain a weighted value for the patch,

$$
v w=\sum_{n=1}^{n=i}\left(Q_{i} * 1 / T_{i}\right) \div \sum_{n=1}^{n=i}\left(1 / T_{i}\right) .
$$

To illustrate, imagine that a forager has only two patches, A and $\mathrm{B}$, and that it finds Patch $\mathrm{A}$ yielding items at a rate of $10 /$ min at 9 a.m., but returning at 10 a.m. finds it temporarily barren, yielding at a rate of $0 / \mathrm{min}$. Traveling directly to the typically leaner patch (B), the forager might obtain items at $3 / \mathrm{min}$. Now, after a 15-min interruption, suppose the animal has the opportunity to resume foraging. Where should it go?

Patch B has been sampled only once, so this single observation receives all the weighting, making its weighted value, $v w b$, the same as its unweighted value, $3 / \mathrm{min}$. However, Patch A was visited twice, the first visit occurring $1.25 \mathrm{~h}$ before, and the second occurring only $0.25 \mathrm{~h}$ earlier. Because of its relative recency, the second experience earns $83 \%$ of the total weighting for Patch A $(1 / .25=4$ and $1 / 1.25=0.8$, so $4 / 4.8=0.833)$, and the first patch experience gets the remainder, $16.7 \%$. Accordingly, $v w a=10 / \mathrm{min} * 0.167=1.67 / \mathrm{min}$. Calculating the value of Patch $\mathrm{B}$ in relation to Patch $\mathrm{A},(V w \mathrm{~B})$, the common equation

$$
V w \mathrm{~B}=v w \mathrm{~b} /(v w \mathrm{a}+v w \mathrm{~b})
$$

is used, where $1 \geq V w \mathrm{~B} \geq 0$, and $V w \mathrm{~A}=1-V w \mathrm{~B}$, revealing that $V w \mathrm{~B}=3 /(3+1.67)=0.64$. Thus Patch $\mathrm{B}$ is still the best bet. Accordingly, it is assumed that the forager returns to Patch B and once again finds the patch yielding 3 items $/ \mathrm{min}$. Suppose the animal is interrupted again, but has another opportunity to forage after $15 \mathrm{~min}$. Now the situation is different, with $v w \mathrm{a}=$ $2.5 / \mathrm{min}$, and $v w \mathrm{~b}$ remaining at $3 / \mathrm{min}$ :

$$
V w \mathrm{~B}=3 /(3+2.5)=0.54 .
$$

The relative value of the two patches is so close to the indifference point, .50 , as to be negligible, so the forager would be about as likely to choose Patch A as Patch B. Using the same computational procedures, if the animal had waited an hour instead of $15 \mathrm{~min}$, the relative value of Patch $B$ would have dropped to .46 , and if it had waited $6 \mathrm{~h}$, Patch B would have been the poorer prospect by far at .39 , which is close to its unweighted relative value, $V u \mathrm{~B}$ :

$$
v u \mathrm{~B}=(3+3) /[(3+3)+(10+0)]=0.38 .
$$

Thus, the higher average value of Patch $A$ reasserts itself with the passage of time, making it the better choice, and spontaneous recovery would almost certainly be observed. That is, the forager would be expected to choose Patch A.

Taking a likely laboratory scenario in which the only "choice" is to respond or not, suppose that a rat is rewarded for running the length of an alley for a few trials and then is given an equal number of extinction trials. Further suppose that the last extinction trial occurred $3 \mathrm{~h}$ after the last rewarded trial. If the animal is tested $15 \mathrm{~min}$ after the second stage of training, the last state of the alley (empty) is 13 times more recent than the first,

$$
(1 / 0.25) /(1 / 3.25)=4 / 0.31=13.0,
$$

and receives $93 \%$ of the weighting, since $4 /(4+0.31) * 100=$ 92.8 , but the second stage of training would be only 1.1 times more recent if the animal is tested after $24 \mathrm{~h}$,

$$
(1 / 24) /(1 / 27)=1.12 \text {, }
$$

where a relative recency of $1.0 \mathrm{implies}$ simultaneity, and therefore the extinction experience would be assigned only $53 \%$ of the weighting. The relative recency of the two outcomes in the alley are virtually contemporaneous after a day's delay, so behavior appropriate to the first stage of training would almost surely emerge because the prospects of reward, though not certain, are vastly improved. An animal's expectations about running the alley at these different points in time are simple functions of the relative recency of training stages, since the same number of trials was used for each. The expectations might be considered to be probabilities or odds that the alley has returned to its rewarded state. For the 15 -min delay, the $93 \%$ likelihood of finding nothing would be unlikely to appeal to most subjects, but the $53 \%$ likelihood should appeal to most. Of course, degree of hunger and other subject variables are always free to operate in settings where the only option is to respond or not. As can be seen in the foraging example given previously, these unspecifiable factors are all but eliminated in choice studies because it is usually safe to assume that animals will prefer the better prospect, irrespective of hunger, timidity, risk aversion, and so on. 
Note that for the sake of clarity, I have introduced several simplifications. For instance, the duration of foraging bouts and trial lengths have been ignored in my examples. In practice, this additional time would be taken into account. Similarly, subjective estimates of recency are not precisely veridical (L. D. Devenport et al., 1997). As with the timing of anticipated events (see, e.g., Mazur, 1994), recency of past events is sensitive to a subjective timing parameter, $k$. Thus, recency $=1 / k T$. The assumption that there is a one-to-one correspondence between objective patch quality (such as capture rate) and sub- jective patch quality is another simplification. Further, there appears to be an interaction between discount rate and magnitude of delayed rewards (Brunner, Gibbon, \& Fairhurst, 1994; Green, Fristoe, \& Myerson, 1994). TWR's predictions may or may not be subject to such interactions.

(Manuscript received July 3, 1997; revision accepted for publication October 15, 1997.) 